\title{
Medication Dispensing
}

National Cancer Institute

\section{Source}

National Cancer Institute. Medication Dispensing. NCI Thesaurus. Code C128533.

The dispensing of a drug to be self-administered by a patient or to be administered by a healthcare provider. 